\title{
MÉTODO PARA ESTABELECIMENTO DE VALORES DE REFERÊNCIA DE QUALIDADE E CLASSES DE USO DA ÁGUA SUPERFICIAL EM MICROBACIAS NO ESTADO DE SANTA CATARINA, BRASIL METHOD FOR ESTABLISHING QUALITY REFERENCE VALUES AND SURFACE WATER USE CLASSES IN WATERSHEDS IN THE STATE OF SANTA CATARINA, BRAZIL
}

\section{Bruna Stedile Ribeiro} Pacheco

Mestre em Ciência e Tecnologia Ambiental, Universidade do Valei do Itajaí (UNIVALI) - Itajaí (SC), Brasil.

\section{Francisco Carlos}

\section{Deschamps}

Doutor em Ciências (Bioquímica), Univali - Itajaí (SC), Brasil.

\section{Endereço para correspondência:}

Bruna Stedile Ribeiro Pacheco Universidade do Valei do Itajaí Rua Uruguai, 458 - Centro CEP 88302-901 - Itajaí (SC), Brasil E-mail: bruna_stedile@hotmail.com

Recebido em: $13 / 02 / 2019$

Aceito em: 20/09/2019

\section{RESUMO}

O conhecimento sobre a qualidade da água por meio da determinação de seus descritores é fundamental para o estabelecimento de políticas de gestão dos recursos hídricos. Neste trabalho, sugere-se um método simples e prático para determinar valores associados aos descritores de qualidade da água. Foram utilizados resultados acumulados ao longo de dez anos de monitoramento da qualidade da água superficial em microbacias do estado de Santa Catarina. Considerando as cinco classes de uso já estabelecidas na legislação federal (Resolução do Conselho Nacional do Meio Ambiente CONAMA no 357/2005), foram determinados os limites para cada classe tendo por base a distribuição dos valores dos parâmetros em estudo. A mediana foi utilizada para estabelecer os valores de referência de qualidade e o percentil 60 como limite máximo para considerar o valor do descritor como aceitável. O método apresentado é de fácil aplicação e os valores calculados, comparados à legislação vigente, são mais restritivos, sugerindo boa qualidade da água.

Palavras-chave: gestão dos recursos hídricos; monitoramento; descritores.

\section{ABSTRACT}

The knowledge about water quality through the determination of its descriptors is fundamental to establish water resource management policies. This work suggests a simple and practical method to determine values associated with water quality descriptors. For this purpose, it was necessary to use the results accumulated over 10 years of surface water quality monitoring from watersheds in Santa Catarina State. Taking into account the five classes of use already established in federal legislation (CONAMA Resolution no 357/2005), the limits for each class were determined based on the distribution of the values of the parameters under study. The median was used to establish the quality reference values and the $60^{\text {th }}$ percentile as the upper limit to consider the descriptor value as acceptable. The method presented is easy to apply, and the calculated values, compared to the current legislation, are more restrictive when it comes to suggesting good water quality.

Keywords: water resource management; monitoring; descriptors. 


\section{INTRODUÇÃO}

A disponibilidade de água em quantidade e qualidade é condição essencial para o desenvolvimento social e econômico de uma região. Se confirmado o aumento do consumo pela diversificação de usos, é razoável supor que a satisfação das duas condições apresentadas estará comprometida. Manter os suprimentos de água com qualidade tem se tornado desafiador, já que o aumento da população e o incremento nas atividades econômicas demandam volumes crescentes de água.

Muitas discussões nessa área são comprometidas pela falta da adequada delimitação do que se pode entender por qualidade da água. A água em seu estado natural tem sua qualidade definida a partir da dissolução de cátions, ânions e sílica, originários de rochas, solos e sedimentos, além de substâncias orgânicas e atividade microbiológica do solo ou do próprio ambiente aquático (ABREU; CUNHA, 2015). Processos naturais podem causar alterações nas características físicas, químicas e microbiológicas da água, tendo consequências adversas para os ecossistemas (SOUZA, 2011; MUNIZ et al., 2011). Entretanto, considera-se que são as intervenções humanas no ambiente natural, independentemente da sua intensidade, as que mais afetam negativamente a qualidade da água (MOSS, 2008).

Desse modo, alterações na qualidade da água podem estar associadas a fatores antrópicos ou naturais. Então, mesmo sem ação humana, a água pode apresentar características indesejáveis que comprometem sua qualidade. Isso complica a avaliação da qualidade da água, especialmente quando se quer buscar fontes de poluição das águas superficiais. Parte dessa limitação está relacionada aos métodos analíticos e às abordagens efetuadas para algumas avaliações (MINELLA; MERTEN, 2011).

Como as variáveis ou descritores que delimitam a qualidade da água são dinâmicos, é fundamental a implantação de programas que monitoram a qualidade dos recursos hídricos ao longo do tempo. É uma atividade essencial na gestão das bacias hidrográficas, para que sejam implementados os instrumentos da política nacional de recursos hídricos, como o planejamento, a gestão, a outorga, a cobrança e o enquadramento dos cursos d'água (TRINDADE et al., 2017). Um dilema, nesse caso, será a escolha das variáveis a serem monitoradas, considerando que é o uso a que se destina a água o que vai determinar as características desejadas. Esse princípio norteou a política de preservação dos recursos hídricos no Brasil, sendo adotados critérios de classificação para o enquadramento dos corpos d'água que levam em consideração seus usos preponderantes (BOLLMANN; MARQUES, 2000; PORTO; PORTO, 2008; COSTA et al., 2014).

No Brasil, muitos trabalhos têm buscado estabelecer metodologias que ofereçam suporte ao enquadramento de corpos d'água superficiais (GUIMARÃES et al., 2016). Análises químicas, físicas e a presença de coliformes ainda são as principais referências para avaliar a qualidade da água, mas as maiores preocupações se voltam àquelas substâncias agregadas aos sistemas hídricos a partir das atividades humanas, como pesticidas, metais pesados, hidrocarbonetos poliaromáticos, desreguladores endócrinos, entre outras. Por outro lado, monitorar recursos hídricos por longos períodos em várias estações amostrais produz um banco de dados muito extenso, gerando certa complexidade na sua interpretação (TRINDADE et al., 2017; ARRUDA; RIZZI; MIRANDA, 2015).

Quando há informações disponíveis e significativas geradas pelo monitoramento, especialmente nos casos de licenciamento ambiental, estas acabam sendo subutilizadas, tendo em vista as extensas e complexas matrizes geradas (ARRUDA; KNOPIK; SOTTOMAIOR, 2017; STROBL; ROBILLARD, 2008). Mesmo que se tenha uma boa base de dados, a escolha do método mais adequado para estabelecer valores de referência ainda pode ser controverso, já que deveria ser simples, de fácil aplicação e possibilidade de reprodução em situações distintas. Ademais, pela grande extensão territorial do país, é impraticável condicionar padrões ambientais nacionalmente uniformes sem considerar as diferenças regionais (MOZEJKO, 2012; MINELLA; MERTEN, 2011; BIEGER et al., 2010; MONDAL; KAVIRAJ; SAHA, 2010; MIGLIACCIO et al., 2007).

O Brasil tem sua legislação relacionada à qualidade da água baseada em diretrizes internacionais, constituindo-se praticamente em cópias delas. Entretanto, estabelecer valores de referência baseados em diretrizes internacionais, sem levar em conta as características locais, pode ter implicações negativas na gestão dos recursos hídricos, pois esses valores não são basea- 
dos na qualidade natural local das águas. De acordo com Pizella e Souza (2007), esse é um problema característico dos países subdesenvolvidos, e acreditase que parte dos motivos sejam os custos envolvidos relacionados à necessidade de amplas informações ambientais e testes laboratoriais.

De acordo com Smith, Alexander e Schwarz (2003), uma abordagem promissora para estimar condições de referência envolve o uso de dados de sistemas pequenos, moderadamente afetados, e a aplicação de técnicas de modelagem estatística para obter referência de valores para grandes rios. Migliaccio et al. (2007) observaram que o comprometimento dos recursos hídricos de microbacias se reflete na bacia hidrográfica como um todo. Entretanto, essa relação será sempre mais evidente dependendo do estágio de degradação da água da microbacia e da diluição a que será submetida quando adicionada ao rio principal que drena a referida bacia.

Portanto, métodos de cálculo simples e bases de dados disponíveis sobre a qualidade da água, independentemente do número de variáveis, seriam uma boa contribuição para melhorar e regionalizar a gestão dos recursos hídricos, seguindo uma tendência que já existe a nível internacional. Como a disponibilidade de séries históricas de dados de qualidade é inviável para todos os cursos d'água, deve-se pelo menos buscar determinar esses parâmetros nos cursos d'água mais representativos do ambiente local.

Assim, o objetivo de produzir Valores de Referência de Qualidade (VRQs) é estabelecer limites que condicionem o equilíbrio entre as necessidades das atividades humanas e a manutenção da capacidade de suporte ambiental, sendo fundamental para identificar alterações pontuais ou sua tendência ao longo do tempo. Esses limites não devem apresentar valores absolutos nem podem ser considerados permanentes, nem mesmo iguais para todo o território nacional, por refletirem características ambientais, desenvolvimento científico e tecnológico e condições econômicas locais e regionais (ABREU; CUNHA, 2015).

Nesse sentido, o presente trabalho teve como objetivo propor critérios e um método de cálculo simples para o estabelecimento de valores de referência e classes de uso baseado em parâmetros de qualidade da água superficial de microbacias no estado de Santa Catarina, cujas atividades econômicas são predominantemente agrícolas. Como forma de ilustrar a aplicabilidade do método proposto, são sugeridos limites para cada parâmetro monitorado para a classificação dos recursos hídricos superficiais em cinco classes de qualidade.

\section{METODOLOGIA}

\section{Área de estudo}

A base de dados utilizada corresponde aos resultados de análise de quatro mil amostras, coletadas entre 1998 e 2008, em 12 microbacias (Figura 1) distribuídas em diferentes regiões do estado de Santa Catarina, abrangendo as cidades de Balneário Camboriú, Camboriú, Schroeder, Lontras, Águas Mornas, Canoinhas, Massaranduba, Luís Alves, Timbó, Sombrio, Palmeira, Ouro e Águas Frias.

O trabalho de monitoramento da qualidade da água nessas microbacias fez parte das atividades referentes ao projeto Microbacias 2 (Programa de Recuperação Ambiental e de Apoio ao Pequeno Produtor Rural), resultante de um acordo entre o estado de Santa Catarina e o Banco Mundial (EPAGRI, 2005). Mesmo não sendo atualizada, a base foi escolhida por abranger mais de 20 parâmetros, cobrir um período de 10 anos, totalizando quatro mil amostras obtidas em diferentes regiões do estado. Há, portanto, boa diversidade de espaço e tempo para garantir a representatividade dos valores apresentados e atender ao objetivo do estudo.

Os parâmetros monitorados, o número de amostras e os períodos considerados para o desenvolvimento dessa metodologia não foram os mesmos para todos os pontos, pois as informações utilizadas foram originadas de resultados das medições de qualidade da água preexistentes, procurando assim fazer aproveitamento total dos dados disponibilizados. Evidencia-se um dos pontos positivos dessa metodologia, pois permite que todos os dados disponíveis sejam utilizados, constituindo-se em uma metodologia aberta e adaptável. 


\section{Parâmetros monitorados e métodos de análise}

Os parâmetros de qualidade da água determinados foram: nitrato, nitrito, oxigênio dissolvido (OD), fosfato-orto, $\mathrm{pH}$, potássio, temperatura, turbidez, alcalinidade, amônia, coliformes termotolerantes e totais, condutividade, ferro, fósforo total, demanda química de oxigênio (DQO), dureza, demanda bioquímica de oxigênio (DBO), sólidos totais, sólidos dissolvidos totais, sólidos voláteis e sólidos suspensos totais. Por sua natureza, as medições de temperatura, pH, condutividade e OD foram realizadas no momento da coleta das amostras diretamente nos corpos d'água, utilizando-se um equipamento de campo da marca Orion modelo 1230. Para as demais determinações, as amostras foram transportadas para os laboratórios de análise de água da Empresa de Pesquisa Agropecuária e Extensão Rural de Santa Catarina (Epagri), localizados nas estações experimentais de Itajaí, Urussanga e Chapecó, conforme a proximidade do local de coleta.

Métodos espectrofotométricos foram utilizados para quantificar amônia, nitrato, nitrito, fosfato-orto, ferro, potássio e fósforo, a partir dos métodos de análise descritos no Standard Methods for the Examination of Water and Wastewater - $20^{\mathrm{a}}$ edição. As leituras foram realizadas em espectrofotômetro FEMTO modelo $600 \mathrm{~S}$. Eventuais aferições foram realizadas em espectrofotômetro Zeiss modelo M500, com duplo feixe. Padrões externos com rastreabilidade - Instituto Nacional de Padrões e Tecnologias (National Institute of Standarts and

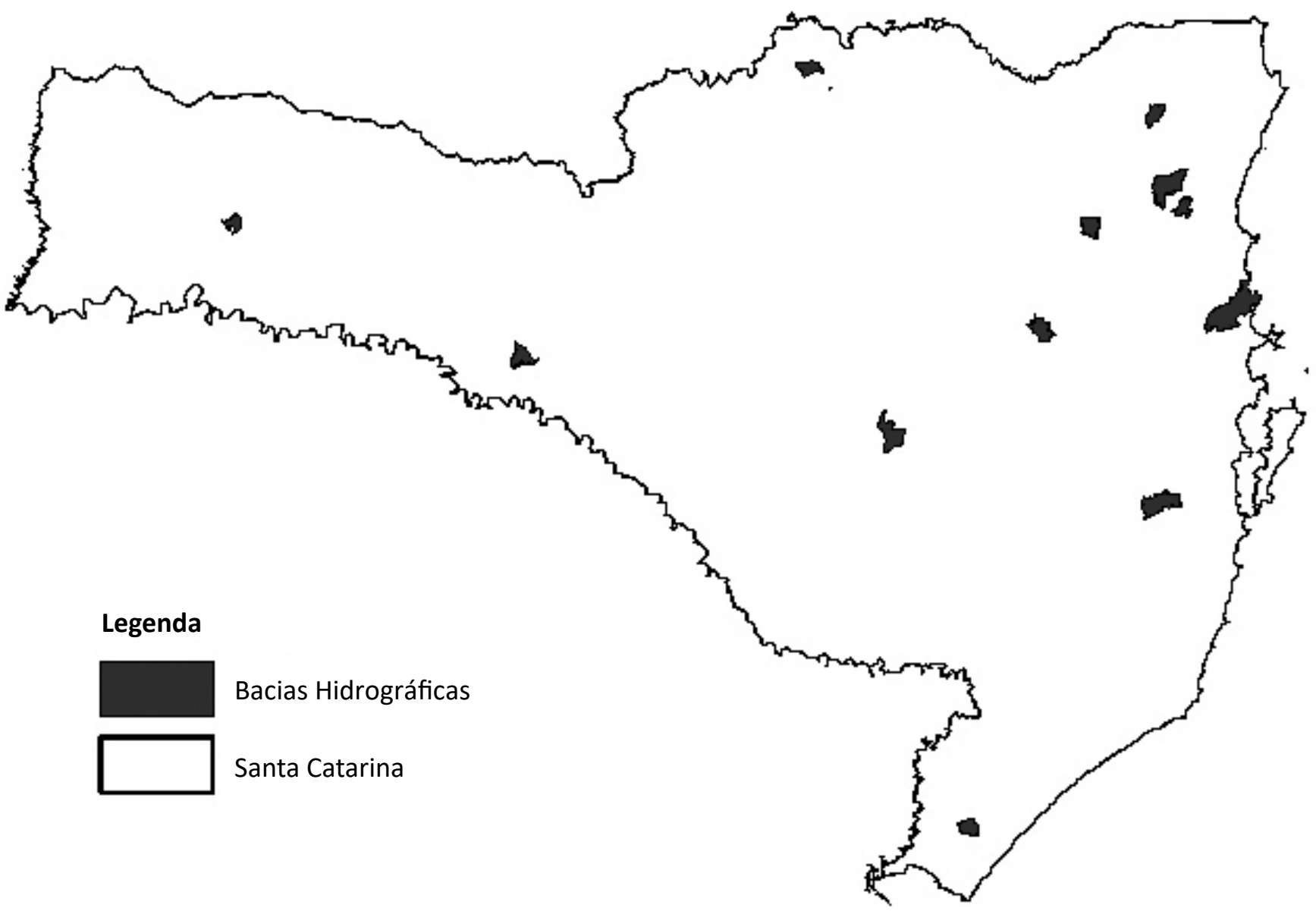

Figura 1 - Localização das microbacias monitoradas no estado de Santa Catarina, Brasil, entre 1998 e 2008. 
Technology - NIST) - foram utilizados para montagem das curvas de calibração. A medida de turbidez foi efetuada por meio do turbidímetro $\mathrm{HACH}$ modelo 2100P. A alcalinidade foi determinada utilizando-se o método da titulação potenciométrica até $\mathrm{pH}$ predeterminado, segundo a norma da Companhia Ambiental do Estado de São Paulo (CETESB) no L5.102 (CETESB, 1992a), enquanto a dureza total foi baseada no método titulométrico do Ácido Etilenodiamino Tetra-Acético (EDTA), segundo a norma CETESB no L5.124 (CETESB, 1992b). A quantificação simultânea de coliformes totais e termotolerantes (Escherichia coli) foi realizada por meio do reativo enzimático Colilert, o qual utiliza nutrientes indicadores que produzem cor e fluorescência ao serem metabolizados por coliformes totais e fecais, quando incubados por 24 horas em estufa a $35 \stackrel{\circ}{\circ}( \pm 0,5 \circ C)$.

\section{Estabelecimento dos valores de referência de qualidade}

A mediana foi escolhida como medida de tendência central para representar o VRQ de cada parâmetro em razão das distorções que o uso da média aritmética pode apresentar como resultado da discrepância de valores a que algumas variáveis estão naturalmente sujeitas. Isso ocorre quando amostras são coletadas em épocas de eventos extremos em que os valores de algumas variáveis se apresentam muito acima daqueles considerados aceitáveis. Considerando que esses valores são de origem natural, é desaconselhável seu descarte do conjunto de dados em avaliação.

A concentração de determinados parâmetros pode variar em coletas realizadas em um mesmo ponto. Essa variação não significa, contudo, uma piora na qua- lidade da água, mas uma variação diária e/ou sazonal normal que pode estar relacionada às condições climáticas, por exemplo, as quais variam em um curto espaço de tempo. Nesse sentido, o percentil 60 foi proposto e adotado para estabelecer o valor máximo "aceitável" de cada parâmetro, eliminando assim possíveis erros analíticos ou de anotação. A escolha desse ponto de corte baseou-se em estudo dos principais métodos que utilizam Índices de Qualidade da Água (IQAs) em que a água é descrita como "aceitável" (AGRIZZI et al., 2018; CECCONELLO; CENTENO; GUEDES, 2018; MENEZES et al., 2016; ARRUDA; RIZZI; MIRANDA, 2015; SABINO; LAGE; ALMEIDA, 2014; MORETTO et al., 2012; SIQUEIRA; APRILE; MIGUÉIS, 2012).

\section{Estabelecimento de classes de uso e respectivos limites}

Para o estabelecimento das classes de qualidade e seus respectivos limites foi desenvolvida uma matriz contendo o intervalo de classe dos parâmetros monitorados obtidos pelos percentis. Essa matriz apresenta uma divisão em cinco classes de qualidade (ótima, boa, aceitável, ruim e péssima), as quais foram estabelecidas a partir de percentis $20,40,60,80$ e 100, respectivamente, determinados para cada parâmetro monitorado. Assim, a classe ótima corresponde ao percentil 20; a classe boa, ao percentil 40; a classe aceitável, ao percentil 60; a classe ruim, ao percentil 80; e a classe péssima, ao percentil 100 . Ressalta-se que valores baixos na concentração de alguns parâmetros indicam boa qualidade. Assim, a classe ótima, por exemplo, abrange os valores mais baixos que compreendem os
$20 \%$ menores da distribuição da base de dados estudada (percentil 20). Ficou fora desse critério o OD, considerando que maiores concentrações representam meIhor qualidade. Já o $\mathrm{pH}$ foi distribuído em faixas, sendo considerada a melhor aquela estabelecida inicialmente em torno da mediana $(6,8)$. Para a temperatura não foram feitas sugestões de classes, uma vez que há variações naturais ao longo do ano e do dia, tornando sem significado prático o estabelecimento de limites, pelo menos no presente contexto.

A sugestão de estabelecer classes de acordo com a concentração de elementos nas amostras foi adotada como forma de reproduzir localmente o que já estabelece a legislação federal (Resolução CONAMA no 357, de 2005).

\section{RESULTADOS E DISCUSSÃO}

Tendo como ponto de partida a base de dados gerada ao longo de dez anos de monitoramento e aplicando-se o método de cálculo proposto no presente traba-
Iho, foi possível estabelecer valores de estatísticas descritivas para todos os parâmetros estudados, incluindo a sugestão para os VRQs (Tabela 1). Usando estatísticas 
simples, o método apresenta-se versátil, independentemente do número de variáveis disponíveis, é de fácil aplicação, não exigindo grande nível de especialização técnica nem sofisticados programas de análise de dados. Estatísticas simples podem apresentar maior utilidade, como no caso do uso da mediana, que se mostrou mais representativa em relação ao uso da média. O caso da turbidez é bastante ilustrativo, já que o valor da mediana foi determinado em 11,1 NTUs (Unidades de Turbidez Nefelométrica), enquanto a média alcançou 26,1 NTUs. Isso é consequência do número elevado de observações cujos valores de turbidez estão acima de 300 NTUs, chegando ao máximo de 984 NTUs (Tabela 1). Entretanto, os valores relatados não representam erro de determinação, sendo resultado dos muitos eventos de chuva que ocorrem naturalmente. Em outros trabalhos relativos à turbidez, houve ampla variação de valores com marcante sazonalidade (ANTUNES et al., 2014; ROSSITER et al., 2015; AMORIM et al., 2017). Dessa forma, a pura eliminação de valores elevados da base de dados não se justifica, já que, como visto, eles podem resultar de eventos naturais.

Uma vez estabelecidos esses pressupostos, é sugestão do presente estudo que o valor do percentil 60, denominado Valor de Prevenção (VP), seja adotado como limite superior admitido. Acima disso, seria indicador de que alguma situação natural extraordinária ou provocada por ação humana ocorreu naquele ambiente, cabendo aos técnicos identificar e avaliar adequadamente o fenômeno.

Para estabelecer limites e associar essas medidas com possíveis usos das águas superficiais, foi publicada uma resolução pelo CONAMA ( $n$ o 357/2005) que atribui cinco classes indicadoras da qualidade da água doce. Aplicando-se essa abordagem no desenvolvimento do presente trabalho, foram determinados, a título ilustrativo, valores limites para cinco classes de uso das águas superficiais de Santa Catarina, apresentados na Tabela 2. Considerando as variáveis estudadas, estabelecer comparações com outros estudos e legislações é desafiador, em parte pelas realidades que representam e pela simples falta de valores sugeridos. Na Tabela 3, são apresentados valores constantes das legislações do país e algumas recomendações internacionais, ressaltando-se que boa parte das variáveis carece de qualquer referência de valor. Dessa forma, os resultados gerados no presente trabalho podem ajudar a ampliar as discussões e novas abordagens sobre a proposição de valores e limites de referência da qualidade da água superficial no país.

Abordagens complexas nem sempre se mostram as mais adequadas e a simplificação pode significar um grande desafio, exigindo um bom roteiro para exploração, abordagem estatística e estimativa de tendências (MOZEJKO, 2012). Diversos são os trabalhos que fazem uso de métodos estatísticos complexos de análise multivariada para avaliação da qualidade da água ou formulação de IQAs, como os apresentados por Agrizzi et al. (2018); Cecconello, Centeno e Guedes (2018); Menezes et al. (2016); Arruda, Rizzi e Miranda (2015); Sabino, Lage e Almeida (2014); Moretto et al. (2012); e Siqueira, Aprile e Miguéis (2012). Como desvantagem, as técnicas de estatística avançada são mais difíceis de aplicar e complexas na interpretação dos resultados, limitações essas que o presente trabalho pretende superar ao propor uma metodologia mais simples de aplicar e interpretar.

Nonato et al. (2007) fazem extenso uso de técnicas multivariadas para determinar um número mínimo de descritores representativos da qualidade da água, buscando reduzir custos de um programa de monitoramento. Mesmo com uma justificativa adequada para a escolha da técnica de análise, esse reducionismo pode eliminar variáveis importantes, comprometendo avaliações ambientais futuras, pela falta dos VRQs da água. Por sua vez, IQAs geralmente apresentam um pequeno número de variáveis, excluindo muitas das que podem ser mais representativas daquele ambiente em estudo. Nesse caso, a técnica escolhida mostra-se adequada para atender aos objetivos estabelecidos, tendo em vista que considera a utilização de todos os dados que estejam disponíveis. Interessante observar que programas de monitoramento bem estruturados, como aqueles conduzidos pela CETESB, buscam ampliar o leque de parâmetros para caracterizar a qualidade da água e efluentes. Em parte, essa foi uma das pressuposições do presente trabalho, o qual priorizou pelo maior número de descritores em análise, com bom espaço de abrangência e repetição ao longo do tempo, aplicando estatísticas simples (Tabela 1).

Nos trabalhos de avaliação da qualidade da água realizados por Amorim et al. (2017), Costa et al. (2014) e Rossiter et al. (2015), por exemplo, os resultados das análises foram confrontados com a Resolução CONAMA 
no 357/2005. Isso foi realizado no presente trabalho, confrontando os valores estabelecidos com os limites da legislação federal, o que permitiu reflexões interessantes. Apesar de a Resolução CONAMA no 357/2005 não possuir valores de concentrações estabelecidos para todas as classes e/ou para todos os parâmetros em estudo, o presente método de cálculo permitiu estabelecer valores para cinco classes de qualidade e para todos os parâmetros que possuíam dados disponíveis pelo monitoramento realizado. Assim, entre os 23 parâmetros estudados,

Tabela 1 - Distribuição dos valores determinados para os diversos parâmetros estudados, considerando a mediana como valor de referência de qualidade (VRQ) e o percentil 60 como valor máximo admitido que ainda reflete o padrão de normalidade, denominado de valor de prevenção (VP).

\begin{tabular}{|c|c|c|c|c|c|}
\hline Parâmetros & Mínimo & Média & $\begin{array}{c}\text { VRQ } \\
\text { mediana }\end{array}$ & $\begin{array}{c}\text { VP } \\
\text { Percentil } 60\end{array}$ & Máximo \\
\hline Alcalinidade $\left(\mathrm{mg} / \mathrm{L}-\mathrm{CaCO}_{3}\right)$ & 0 & 33,35 & 32,00 & 34,00 & 148,00 \\
\hline Amônia (mg/L-N) & 0 & 1,35 & 1,19 & 1,50 & 10,31 \\
\hline Cálcio (mg/L) & 0,04 & 1,50 & 0,92 & 1,12 & 8,00 \\
\hline Coliformes termotolerantes (NMP) & 0 & 3869 & 980 & 1560 & 241920 \\
\hline Coliformes totais (NMP) & 0 & 47747 & 24192 & 30760 & 241920 \\
\hline Condutividade elétrica (uS/cm) & 2,20 & 69,74 & 64,00 & 70,00 & 1416,00 \\
\hline $\mathrm{DBO}(\mathrm{mg} / \mathrm{L})$ & 0 & 1,98 & 1,11 & 1,60 & 85,00 \\
\hline $\mathrm{DQO}(\mathrm{mg} / \mathrm{L})$ & 0 & 17,58 & 7,90 & 10,78 & 566,04 \\
\hline Dureza (mg/L) & 0 & 24,17 & 22,00 & 24,00 & 152,00 \\
\hline Ferro (mg/L) & 0 & 0,86 & 0,63 & 0,83 & 13,49 \\
\hline Fósforo total (mg/L-P) & 0 & 0,18 & 0,14 & 0,16 & 6,57 \\
\hline Fosfato-orto (mg/L-P) & 0 & 0,09 & 0,07 & 0,08 & 2,14 \\
\hline Magnésio (mg/L) & 0,03 & 1,40 & 1,00 & 1,24 & 29,84 \\
\hline Nitrato (mg/L-N) & 0 & 0,60 & 0,26 & 0,37 & 8,76 \\
\hline Nitrito (mg/L-N) & 0 & 0,02 & 0,01 & 0,02 & 1,55 \\
\hline $\mathrm{OD}(\mathrm{mg} / \mathrm{L})$ & 0,07 & 8,35 & 8,44 & 8,70 & 12,63 \\
\hline Potencial hidrogeniônico $(\mathrm{pH})$ & 4,68 & 6,83 & 6,80 & 6,96 & 9,20 \\
\hline Potássio (mg/L) & 0 & 2,22 & 2,00 & 2,30 & 27,00 \\
\hline Sólidos dissolvidos totais (mg/L) & 12 & 96 & 73 & 80 & 1889 \\
\hline Sólidos suspensos totais (mg/L) & 1 & 51 & 30 & 40 & 889 \\
\hline Sólidos totais (mg/L) & 32 & 145 & 104 & 114 & 1967 \\
\hline Sólidos voláteis (mg/L) & 3 & 44 & 34 & 41 & 220 \\
\hline Turbidez (NTU) & 0,07 & 26,10 & 11,10 & 13,80 & 984,00 \\
\hline
\end{tabular}

NMP: número mais que provável; DBO: demanda bioquímica de oxigênio; DQO: demanda química de oxigênio; OD: oxigênio dissolvido; NTU: unidade de turbidez nefelométrica. 
apenas 11 possuíam concentrações limites estabelecidos pela Resolução CONAMA no 357/2005: nitrato, nitrito, DBO, OD, pH, turbidez, amônia, coliformes termotolerantes, ferro, fósforo total e sólidos dissolvidos totais (SDT).
A legislação brasileira é baseada em diretrizes internacionais, as quais consideram critérios científicos e estudos toxicológicos para preservação da vida aquática e saúde humana. Os critérios científicos internacionais

Tabela 2 - Valores limites sugeridos pelo presente trabalho para os parâmetros que descrevem a qualidade das diferentes classes de águas superficiais (doce) em Santa Catarina, Brasil.

\begin{tabular}{|c|c|c|c|c|c|c|}
\hline Classificação & Ótima & Boa & Aceitável & Ruim & Péssima & $\begin{array}{c}\text { CONAMA no } \\
357 / 2005\end{array}$ \\
\hline Percentis & 20 & 40 & 60 & 80 & 100 & - \\
\hline $\begin{array}{l}\text { Classe - Resolução } \\
\text { CONAMA no 357/2005 }\end{array}$ & $\begin{array}{l}\text { Classe } \\
\text { especial }\end{array}$ & Classe 1 & Classe 2 & Classe 3 & Classe 4 & Classe 2 \\
\hline Alcalinidade $\left(\mathrm{mg} / \mathrm{L}-\mathrm{CaCO}_{3}\right)$ & 22,00 & 30,00 & 34,00 & 42,00 & 148,00 & - \\
\hline Amônia (mg/L-N) & 0,32 & 0,85 & 1,50 & 2,17 & 10,31 & $4,5^{*}$ \\
\hline Cálcio (mg/L) & 0,35 & 0,70 & 1,12 & 2,28 & 8,00 & - \\
\hline Coliformes termotolerantes (NMP) & 200 & 630 & 1560 & 3558 & 241920 & 1000 \\
\hline Coliformes totais (NMP) & 8098 & 19180 & 30760 & 68670 & 241920 & - \\
\hline Condutividade elétrica (uS/cm) & 49,00 & 59,00 & 70,00 & 84,90 & 1416,00 & - \\
\hline $\mathrm{DBO}(\mathrm{mg} / \mathrm{L})$ & 0,35 & 0,86 & 1,60 & 3,00 & 85,00 & 5,0 \\
\hline $\mathrm{DQO}(\mathrm{mg} / \mathrm{L})$ & 3,09 & 6,00 & 10,78 & 26,78 & 566,04 & - \\
\hline Dureza (mg/L) & 14,00 & 20,00 & 24,00 & 32,00 & 152,00 & - \\
\hline Ferro (mg/L) & 0,34 & 0,58 & 0,83 & 1,41 & 13,49 & 0,3 \\
\hline Fósforo total (mg/L-P) & 0,08 & 0,12 & 0,16 & 0,24 & 6,57 & 0,1 \\
\hline Fósfato-orto (mg/L-P) & 0,02 & 0,06 & 0,08 & 0,11 & 2,14 & - \\
\hline Magnésio (mg/L) & 0,57 & 0,86 & 1,24 & 1,72 & 29,84 & - \\
\hline Nitrato (mg/L-N) & 0,09 & 0,19 & 0,37 & 0,86 & 8,76 & 10,0 \\
\hline Nitrito (mg/L-N) & 0,001 & 0,009 & 0,02 & 0,03 & 1,55 & 1,0 \\
\hline Potássio (mg/L) & 1,10 & 1,70 & 2,30 & 3,00 & 27,00 & - \\
\hline Sólidos dissolvidos totais (mg/L) & 57 & 67 & 80 & 92 & 1889 & 500,0 \\
\hline Sólidos suspensos totais (mg/L) & 9 & 21 & 40 & 71 & 889 & - \\
\hline Sólidos totais (mg/L) & 81 & 97 & 114 & 151 & 1967 & - \\
\hline Sólidos voláteis (mg/L) & 18 & 28 & 41 & 66 & 220 & - \\
\hline Turbidez (NTU) & 4,31 & 8,93 & 13,80 & 26,02 & 984,00 & - \\
\hline $\mathrm{OD}(\mathrm{mg} / \mathrm{L})$ & $>7,52$ & $>8,20$ & $>8,70$ & $>9,25$ & $>12,63$ & $>5,0$ \\
\hline
\end{tabular}

CONAMA: Conselho Nacional do Meio Ambiente; NMP: número mais que provável; DBO: demanda bioquímica de oxigênio; DQO: demanda química de oxigênio; NTU: Unidade de turbidez nefelométrica; OD: Oxigênio dissolvido; *para pH menor ou igual a 7,5 (CONAMA, 2005). 
Tabela 3 - Valores de referência para algumas variáveis disponíveis na legislação do Brasil e algumas referências complementares.

\begin{tabular}{|c|c|c|c|c|c|c|c|c|c|}
\hline \multirow{2}{*}{\multicolumn{2}{|c|}{$\begin{array}{l}\text { Variáveis } \\
\text { Unidade }\end{array}$}} & $\mathrm{Fe}$ & $\mathbf{P}$ & Mg & $\mathrm{NO}^{-3}$ & $\mathrm{NO}^{-2}$ & \multirow{2}{*}{ pH } & SDT & $\mathbf{T}$ \\
\hline & & $\mathrm{mg} / \mathrm{L}$ & $\mathrm{mg} / \mathrm{L}$ - P & $\mathrm{mg} / \mathrm{L}$ & $\mathrm{mg} / \mathrm{L}-\mathbf{N}$ & $\mathrm{mg} / \mathrm{L}-\mathrm{N}$ & & $\mathrm{mg} / \mathrm{L}$ & NTU \\
\hline \multirow{5}{*}{$\begin{array}{l}\text { Resolução } \\
\text { CONAMA } \\
\text { no } \\
357 / 2005\end{array}$} & $\begin{array}{l}\text { Classe } \\
\text { especial }\end{array}$ & $\begin{array}{l}\text { condições } \\
\text { naturais }\end{array}$ & $\begin{array}{c}\text { condições } \\
\text { naturais }\end{array}$ & $\begin{array}{c}\text { condições } \\
\text { naturais }\end{array}$ & $\begin{array}{c}\text { condições } \\
\text { naturais }\end{array}$ & $\begin{array}{c}\text { condições } \\
\text { naturais }\end{array}$ & $\begin{array}{l}\text { condições } \\
\text { naturais }\end{array}$ & $\begin{array}{c}\text { condições } \\
\text { naturais }\end{array}$ & $\begin{array}{c}\text { condições } \\
\text { naturais }\end{array}$ \\
\hline & Classe 1 & 0,3 & 0,1 & n.s & 10 & 1 & 6 a 9 & 500 & 40 \\
\hline & Classe 2 & 0,3 & 0,1 & n.s & 10 & 1 & 6 a 9 & 500 & 100 \\
\hline & Classe 3 & 5 & 0,15 & n.s & 10 & 1 & 6 a 9 & n.s & 100 \\
\hline & Classe 4 & n.s & n.s & n.s & n.s & n.s & 6 a 9 & n.s & n.s \\
\hline \multirow{4}{*}{$\begin{array}{l}\text { Decreto no } \\
14.250 / 1981\end{array}$} & Classe 1 & $\begin{array}{c}\text { sem } \\
\text { lançamento }\end{array}$ & $\begin{array}{c}\text { sem } \\
\text { lançamento }\end{array}$ & $\begin{array}{c}\text { sem } \\
\text { lançamento }\end{array}$ & $\begin{array}{c}\text { sem } \\
\text { lançamento }\end{array}$ & $\begin{array}{c}\text { sem } \\
\text { lançamento }\end{array}$ & $\begin{array}{c}\text { sem } \\
\text { lançamento }\end{array}$ & $\begin{array}{c}\text { sem } \\
\text { lançamento }\end{array}$ & $\begin{array}{c}\text { sem } \\
\text { lançamento }\end{array}$ \\
\hline & Classe 2 & n.s & n.s & n.s & 10 & 1 & n.s & n.s & n.s \\
\hline & Classe 3 & n.s & n.s & n.s & 10 & 1 & n.s & n.s & n.s \\
\hline & Classe 4 & n.s & n.s & n.s & 10 & 1 & n.s & n.s & n.s \\
\hline \multirow{4}{*}{$\begin{array}{l}\text { Resolução } \\
\text { CONAMA } \\
\text { no } \\
396 / 2008\end{array}$} & $\begin{array}{l}\text { Consumo } \\
\text { humano }\end{array}$ & 0,3 & n.s & n.s & 10 & 1 & n.s & 1000 & n.s \\
\hline & $\begin{array}{c}\text { Dessedentação } \\
\text { animal }\end{array}$ & n.s & n.s & n.s & 90 & 10 & n.s & n.s & n.s \\
\hline & Irrigação & 5 & n.s & n.s & n.s & 1 & n.s & n.s & n.s \\
\hline & Recreação & 0,3 & n.s & n.s & 10 & 1 & n.s & n.s & n.s \\
\hline $\begin{array}{l}\text { Portaria } n= \\
2.914 / 2011\end{array}$ & Potabilidade & 2,4 ou 0,3 & n.s & n.s & 10 & 1 & n.s & 1000 & 5 \\
\hline OMS & $\begin{array}{c}\text { Saúde } \\
\text { humana }\end{array}$ & n.s & n.s & 0,4 & 11 & 0,9 & n.s & n.s & 1 \\
\hline EPA & Vida aquática & 1 & n.s & n.s & n.s & n.s & 6,5 a 9 & n.s & n.s \\
\hline \multirow{3}{*}{ CCME } & $\begin{array}{c}\text { Dessedentação } \\
\text { animal }\end{array}$ & n.s & n.s & n.s & 100 & 10 & n.s & 3000 & n.s \\
\hline & Irrigação & 5 & n.s & n.s & n.s & n.s & n.s & n.s & n.s \\
\hline & Recreação & n.s & n.s & n.s & n.s & n.s & 5 a 9 & n.s & 50 \\
\hline \multicolumn{2}{|l|}{ Variáveis } & Alcalinidade & $\mathrm{NH}_{3}$ & $\mathrm{Ca}$ & CT & C. Term. & DBO & OD & Dureza \\
\hline \multicolumn{2}{|l|}{ Unidade } & $\begin{array}{l}\mathrm{mg} / \mathrm{L}- \\
\mathrm{CaCO}_{3}\end{array}$ & $\mathrm{mg} / \mathrm{L}-\mathrm{NH}_{3}$ & $\mathrm{mg} / \mathrm{L}$ & NMP & NMP & $\mathrm{mg} / \mathrm{L}$ & $\mathrm{mg} / \mathrm{L}$ & $\mathrm{mg} / \mathrm{L}$ \\
\hline \multirow{5}{*}{$\begin{array}{l}\text { Resolução } \\
\text { CONAMA } \\
\text { no } \\
357 / 2005\end{array}$} & $\begin{array}{c}\text { Classe } \\
\text { especial }\end{array}$ & $\begin{array}{l}\text { condições } \\
\text { naturais }\end{array}$ & $\begin{array}{c}\text { condições } \\
\text { naturais }\end{array}$ & $\begin{array}{c}\text { condições } \\
\text { naturais }\end{array}$ & $\begin{array}{l}\text { condições } \\
\text { naturais }\end{array}$ & $\begin{array}{c}\text { condições } \\
\text { naturais }\end{array}$ & $\begin{array}{l}\text { condições } \\
\text { naturais }\end{array}$ & $\begin{array}{c}\text { condições } \\
\text { naturais }\end{array}$ & $\begin{array}{c}\text { condições } \\
\text { naturais }\end{array}$ \\
\hline & Classe 1 & n.s & $*$ & n.s & n.s & 200 & 3 & $>6$ & n.s \\
\hline & Classe 2 & n.s & $*$ & n.s & n.s & 1000 & 5 & $>5$ & n.s \\
\hline & Classe 3 & n.s & $*$ & n.s & n.s & 4000 & 10 & $>4$ & n.s \\
\hline & Classe 4 & n.s & n.s & n.s & n.s & n.s & n.s & $>2$ & n.s \\
\hline
\end{tabular}


Tabela 3 - Continuação.

\begin{tabular}{|c|c|c|c|c|c|c|c|c|c|}
\hline \multicolumn{2}{|l|}{ Variáveis } & Alcalinidade & $\mathrm{NH}_{3}$ & $\mathrm{Ca}$ & CT & C. Term. & DBO & OD & Dureza \\
\hline \multicolumn{2}{|l|}{ Unidade } & $\begin{array}{l}\mathrm{mg} / \mathrm{L}- \\
\mathrm{CaCO}_{3}\end{array}$ & $\mathrm{mg} / \mathrm{L}-\mathrm{NH}_{3}$ & $\mathrm{mg} / \mathrm{L}$ & NMP & NMP & $\mathrm{mg} / \mathrm{L}$ & $\mathrm{mg} / \mathrm{L}$ & $\mathrm{mg} / \mathrm{L}$ \\
\hline \multirow{4}{*}{$\begin{array}{l}\text { Decreto no } \\
14.250 / 1981\end{array}$} & Classe 1 & $\begin{array}{c}\text { sem } \\
\text { lançamento }\end{array}$ & $\begin{array}{c}\text { sem } \\
\text { lançamento }\end{array}$ & $\begin{array}{c}\text { sem } \\
\text { lançamento }\end{array}$ & $\begin{array}{c}\text { sem } \\
\text { lançamento }\end{array}$ & $\begin{array}{c}\text { sem } \\
\text { lançamento }\end{array}$ & $\begin{array}{c}\text { sem } \\
\text { lançamento }\end{array}$ & $\begin{array}{c}\text { sem } \\
\text { lançamento }\end{array}$ & $\begin{array}{c}\text { sem } \\
\text { lançamento }\end{array}$ \\
\hline & Classe 2 & n.s & 0,5 & n.s & 5000 & 1000 & 5 & $>5$ & n.s \\
\hline & Classe 3 & n.s & 0,5 & n.s & 20000 & 4000 & 10 & $>4$ & n.s \\
\hline & Classe 4 & n.s & n.s & n.s & 20000 & 4000 & n.s & $>0,5$ & n.s \\
\hline \multirow{4}{*}{$\begin{array}{l}\text { Resolução } \\
\text { CONAMA } \\
\text { no } \\
396 / 2008\end{array}$} & $\begin{array}{l}\text { Consumo } \\
\text { humano }\end{array}$ & n.s & n.s & n.s & n.s & n.s & n.s & n.s & n.s \\
\hline & $\begin{array}{c}\text { Dessedentação } \\
\text { animal }\end{array}$ & n.s & n.s & n.s & n.s & n.s & n.s & n.s & n.s \\
\hline & Irrigação & n.s & n.s & n.s & n.s & n.s & n.s & n.s & n.s \\
\hline & Recreação & n.s & n.s & n.s & n.s & n.s & n.s & n.s & n.s \\
\hline $\begin{array}{l}\text { Portaria no } \\
2.914 / 2011\end{array}$ & Potabilidade & n.s & n.s & n.s & 0 & 0 & n.s & n.s & 500 \\
\hline OMS & $\begin{array}{c}\text { Saúde } \\
\text { humana }\end{array}$ & n.s & n.s & n.s & 0 & 0 & n.s & n.s & n.s \\
\hline EPA & Vida aquática & 20 & $* *$ & n.s & n.s & n.s & n.s & 3,5 a 9,5 & n.s \\
\hline \multirow{3}{*}{ CCME } & $\begin{array}{c}\text { Dessedentação } \\
\text { animal }\end{array}$ & n.s & n.s & 1000 & n.s & n.s & n.s & n.s & n.s \\
\hline & Irrigação & n.s & n.s & 1000 & n.s & 100 & n.s & n.s & n.s \\
\hline & Recreação & n.s & n.s & n.s & n.s & $\begin{array}{l}200\left(1^{a}\right) \\
1000\left(2^{a}\right)\end{array}$ & n.s & n.s & n.s \\
\hline
\end{tabular}

Fe: ferro; P: fósforo; Mg: magnésio; NO-3: nitrato; NO-2: nitrito; SDT: sólidos dissolvidos totais; T: turbidez; NTU: unidade de turbidez nefelométrica; CONAMA: Conselho Nacional do Meio Ambiente; OMS: Organização Mundial da Saúde; EPA: Environmental Protection Agency; CCME: Canadian Council of Ministers of the Environment; $n$.s: não sugerido pela legislação; $\mathrm{NH}_{3}$ : amônia; $\mathrm{Ca}$ : cálcio; $\mathrm{CT}$ : coliformes totais; $\mathrm{C}$. Term.: coliformes termotolerantes; $\mathrm{DBO}$ : demanda bioquímica de oxigênio; OD: oxigênio dissolvido; $\mathrm{CaCO}_{3}$ : carbonato de cálcio; NMP: número mais que provável; *varia com $\mathrm{pH} ;{ }^{* *}$ varia de espécie para espécie.

geralmente são estudados em diferentes espécies de animais, relacionando o efeito da concentração de um constituinte e uma espécie em particular. A concentração máxima definida é a maior concentração em que não foi observado efeito agudo e/ou crônico, permitindo a exposição por tempo indeterminado, ou seja, o consumo é seguro por toda a vida de consumo. Esses valores, quando extrapolados para saúde humana, geralmente são divididos por um fator de incerteza para segurança. As diretrizes internacionais mais utilizadas para derivações de normas de regulação encontram-se na Organização Mundial da Saúde - OMS (WHO,
2011), para proteção da saúde humana; na Agência de Proteção Ambiental dos Estados Unidos (United States Environmental Protection Agency - USEPA) (1986; 2013), para proteção da vida aquática; e no Conselho Canadense de Ministros do Meio Ambiente (Canadian Council of Ministers of the Environment - CCME) (1993; 1999b), para proteção dos usos: dessedentação animal, irrigação e recreação. Dos 23 parâmetros monitorados neste trabalho, apenas seis estavam estabelecidos pela OMS para saúde humana (coliformes totais e termotolerantes, magnésio, nitrato, nitrito e turbidez); quatro estavam estabelecidos pela USEPA 
para proteção da vida aquática (alcalinidade, ferro, $O D$ e $\mathrm{pH}$ ); e quatro estavam estabelecidas pelo CCME para dessedentação animal (coliformes termotolerantes, nitrato, nitrito e SDT), quatro para irrigação (coliformes termotolerantes, coliformes totais, cálcio e ferro) e três para recreação (coliformes termotolerantes, $\mathrm{pH}$ e turbidez). Na Tabela 3, podem ser visualizados os parâmetros que possuem concentrações associadas em cada referência utilizada.

A maior parte dos trabalhos de monitoramento da qualidade da água utiliza a classe 2 da Resolução CONAMA no 357/2005 como referência para discussão dos resultados. Já para aqueles cursos d'água sem estudo de enquadramento, a classe 2 deve ser estabelecida como referência "provisória". No presente trabalho, foram comparados os valores da classe 2 da Resolução CONAMA no 357/2005 com aqueles determinados no que foi aqui chamado de classe aceitável (percentil 60). Pode-se observar que parâmetros como amônia, nitrato, nitrito, DBO, sólidos dissolvidos totais e turbidez, apresentam-se bem abaixo dos limites da legislação vigente (Tabela 2). Já parâmetros como ferro, fósforo e coliformes termotolerantes apresentaram-se acima dos limites impostos pela legislação, ou seja, apresentaram-se menos restritivos. Esse resultado de incompatibilidades para esses parâmetros também pode ser observado em diversos trabalhos. Gama et al. (2010) estabeleceram concentrações limites para alguns parâmetros em cada classe, sendo que os valores limites estabelecidos para condutividade neste trabalho se assemelharam bastante aos estipulados por esses autores, ao contrário dos valores de coliformes totais, que, neste trabalho, foram menos restritivos.

Variáveis como fósforo, coliformes, turbidez e sólidos totais apresentam oscilações de valores e podem ser bons indicadores de ações antrópicas locais e eventos climáticos, como já relatado em outros estudos, como os de Arruda, Knopik e Sottomaior (2017), Moretto et al. (2012) e Zucco et al. (2012). Essas mesmas variáveis comprometeram a qualidade da água no estudo para o enquadramento legal do Rio Juco (rio de abastecimento de Vitória, Espírito Santo). Essa bacia apresenta relevantes núcleos de atividades agropecuárias, além de vários aglomerados urbanos desprovidos de sistema de tratamento de esgoto (GUIMARÃES et al., 2016), situação semelhante às microbacias monitoradas em Santa Catarina.
Quanto ao parâmetro turbidez, a OMS (WHO, 2011) estabelece a concentração limite de 1 NTU para turbidez, sendo que o ideal seria menor que 0,5 NTU. Essa concentração estabelecida é bem mais restritiva que a classe ótima estabelecida neste trabalho, por exemplo. Entretanto, o CCME (1993), para o uso recreação, estabelece a concentração máxima de 50 NTUs, sendo bem mais "permissiva" que o estabelecido neste trabalho.

Em geral, pode-se observar que os limites dos parâmetros estabelecidos neste trabalho para cada classe foram significativamente menores em relação aos estabelecidos pela Resolução CONAMA no 357/2005 e pelas diretrizes internacionais. Entretanto, fósforo, ferro e coliformes termotolerantes merecem atenção.

Os resultados de incompatibilidades para fósforo podem ser observados em diversos trabalhos, e sua mobilidade pode estar associada a ações antrópicas e naturais (OLIVEIRA; SANTOS; LIMA, 2017; VON SPERLING; CHERNICHARO, 2002). Oliveira, Santos e Lima (2017), Bucci e Oliveira (2014) e Alves et al. (2008) relataram o fósforo como um dos parâmetros mais críticos. Em contraste, Rossiter et al. (2015), no diagnóstico da qualidade da água no canal do Sertão Alagoano, observaram que todos os resultados atenderam às especificações da classe 1 da Resolução CONAMA no 357/2005, com exceção do fósforo total, que exibiu valores de enquadramento relativos à classe 4. Trindade et al. (2017), no monitoramento da qualidade da água na bacia do Rio das Velhas, utilizando uma base de dados de nove anos e com 11 variáveis monitoradas, detectaram dados estáveis com maiores alterações relacionadas a coliformes termotolerantes, DBO e nitrato, principalmente nas regiões próximas a centros urbanos. Sabino, Lage e Noronha (2017), utilizando uma série temporal, com dados de 1998 a 2014, de monitoramento no Córrego Gameleiras, concluíram que o fósforo e o OD ultrapassaram os limites da legislação em $100 \%$ das observações.

Uma revisão no valor apresentado pela legislação, no sentido de adequar à realidade brasileira, seria recomendável, já que a geologia local também pode influenciar na concentração desse elemento na água (fósforo), além de outras condições naturais. Esse é um ponto que o presente trabalho pode contribuir, por estar baseado em ampla amostragem representativa de diferentes regiões de Santa Catarina. 
Já é de longa data a sugestão feita pela USEPA (1986), segundo a qual a definição de valores máximos permitidos para nutrientes, como fósforo e nitrogênio total, deve ser realizada para cada região específica, já que esses parâmetros fornecem informações sobre a qualidade natural das águas do local. O mesmo órgão recomenda a utilização de séries históricas de 10 a 25 anos, selecionando a condição de referência por meio da escolha de locais que representem as águas menos impactadas antropicamente. A presente base de dados, ao cobrir o período de dez anos, atinge esse critério, então. A instituição recomenda pelo menos o uso do percentil 25 para que o valor limite de determinada variável apresente qualidade ótima ou excelente. Ou seja, ao se ordenar os resultados de forma crescente, o valor determinado pelo percentil 25 é que seria o limite dessa classe. Como forma de melhor ilustrar a viabilidade e a versatilidade do método apresentado no presente trabalho, foi escolhido o valor limite dado pelo percentil 20 para representar a classe ótima, ou seja, a melhor condição existente. Cabe observar que esse limite não é impositivo e pode ser alterado quando o método for aplicado em outros estudos a partir de novos critérios ou argumentos apresentados por seus autores. O mérito da presente proposta é que o método não muda em função do critério adotado e qualquer base de dados poderá ser analisada novamente a qualquer momento.

Além do fósforo (limite de $0,2 \mathrm{mg} / \mathrm{L}$, estabelecido para classe aceitável neste trabalho), o ferro pode ter apresentado concentrações elevadas neste trabalho (limite de $0,8 \mathrm{mg} / \mathrm{L}$, estabelecido para a classe aceitável neste trabalho) em razão das condições naturais, por exemplo, dissolução de compostos do solo, ou também pode ter origem antrópica, por exemplo, despejos industriais. O ferro tem pouco significado sanitário nas concentrações usualmente encontradas nas águas naturais. Em pequenas concentrações causam problemas de cor na água, e em certas concentrações podem causar sabor e odor (VON SPERLING; CHERNICHARO, 2002). É bem possível que a concentração elevada desse parâmetro, encontrada nos dados de monitoramento em Santa Catarina, seja em razão das condições naturais, como a composição geológica e pedológica da região. Ademais, em épocas de alta precipitação, o nível de ferro na água pode aumentar em decorrência dos processos de erosão nas margens dos corpos d’água (BRAGA; HESPANHOL, 2005).
Segundo a OMS (WHO, 2011), o ferro é um dos metais mais abundantes da crosta terrestre e é encontrado em águas naturais, variando de 0,5 a $50 \mathrm{mg} \cdot \mathrm{L}^{-1}$. Como precaução contra ingestão excessiva de ferro, foi estabelecida a quantidade máxima de $0,8 \mathrm{mg} \cdot \mathrm{Kg}^{-1} \mathrm{de}$ peso corporal que se aplica para o ferro em todas as fontes, exceto óxidos de ferro usados como corantes e suplementos. A concentração de $0,3 \mathrm{mg} \cdot \mathrm{L}^{-1}$ é estabelecida pela OMS com a finalidade de não afetar o sabor e a aparência da água. De acordo com a USEPA (1986), a concentração máxima de ferro para a proteção da vida aquática é de $1 \mathrm{mg} \cdot \mathrm{L}^{-1}$, e de acordo com o CCME (1999b), para o uso irrigação, a concentração deve ser, no máximo, de $5 \mathrm{mg} \cdot \mathrm{L}^{-1}$.

Quanto aos coliformes termotolerantes, o valor limite estabelecido na classe 2 pela legislação federal (1.000 $\mathrm{NMP}$ - número mais que provável) é bastante inferior ao determinado neste trabalho (1.560 NMP). O CCME (1999b), para o uso irrigação, estabelece o valor máximo de $100 \mathrm{NMP} / 100 \mathrm{~mL}$, para coliformes termotolerantes. Com relação ao uso recreação de contato primário, o CCME estabelece o valor máximo de 200 NMP/100 $\mathrm{mL}$, e para recreação de contato secundário, 1.000 $\mathrm{NMP} / 100 \mathrm{~mL}$. Esses valores são estabelecidos pela média geométrica para no mínimo cinco amostras. Para amostra individual para o uso recreação de contato primário, o valor passa para $400 \mathrm{NMP} / 100 \mathrm{~mL}$. A OMS (WHO, 2011) também recomenda ausência de coliformes totais e termotolerantes em $100 \mathrm{~mL}$. Dificilmente os recursos hídricos naturais sem tratamento apresentarão ausência de coliformes termotolerantes e coliformes totais, por isso toda água destinada para consumo humano deve passar por, no mínimo, desinfecção. O CCME (1999b), para o uso irrigação, estabelece o valor máximo de $100 \mathrm{NMP} / 100 \mathrm{~mL}$ para coliformes termotolerantes e de $1.000 \mathrm{NMP} / 100 \mathrm{~mL}$ para coliformes totais.

Coliformes são indicadores típicos do estado de saneamento da região, tanto em áreas urbanas quanto em áreas rurais, refletindo bem as possíveis intervenções humanas, ou a falta delas. Os conglomerados urbanos precisam de água em abundância para sustentar as inúmeras atividades ali desenvolvidas. Sem os devidos processos mitigatórios, a água tende a retornar ao ambiente invariavelmente com sua qualidade comprometida. No documento Atlas Esgotos, da Agência Nacional de Águas (ANA, 2017), encontra-se uma descrição nada animadora sobre esse tema. Apenas para 
ilustrar, cita-se que $27 \%$ da população é desprovida de qualquer atendimento de coleta e tratamento de esgoto. Deve-se considerar ainda que as áreas rurais carecem de instrumentos legais e técnicos que diminuam os efeitos negativos de cargas orgânicas de origem doméstica ou animal sobre as águas superficiais. Mesmo na atualidade, o saneamento rural ainda está na dependência das taxas de autodepuração dos recursos hídricos que banham a região, podendo, em parte, explicar os valores mais elevados de coliformes determinados no presente trabalho.

Felizmente, baseado no que foi observado no presente estudo, parece que a qualidade da água superficial da área de abrangência do estudo está razoavelmente preservada. Os resultados de qualidade obtidos geram então um novo desafio, que é a manutenção da integridade dos recursos hídricos do estado, frente às crescentes demandas. Para tanto, é necessário adotar políticas públicas que evitem o incremento de poluentes nos recursos hídricos, incluindo aí o cumprimento de metas estabelecidas nos objetivos do desenvolvimento sustentável. Em países de grandes dimensões como o
Brasil, o ideal seria que cada estado ou mesmo região de abrangência de cada bacia hidrográfica estabelecesse seus próprios valores de referência. Para tanto, seriam necessários longos e abrangentes estudos de monitoramento da qualidade da água, ou mesmo novas tecnologias que permitissem estabelecer os valores de referência. Assim, com a adaptação desses limites nas bacias hidrográficas, a gestão dos recursos hídricos poderia ser favorecida, promovendo constantes aprimoramentos na legislação e nas ações preventivas ou mitigatórias (VON SPERLING; CHERNICHARO, 2002).

Considerando a diversidade cultural e as restrições econômicas do país, a metodologia apresentada é uma contribuição efetiva para simplificar e flexibilizar a forma de estabelecer os valores de referência e os limites de classes dos recursos hídricos, sem contrapor a política de recursos hídricos já estabelecida. A metodologia desenvolvida pode ainda facilitar os estudos sobre o enquadramento de recursos hídricos, tendo em vista que cria uma linguagem acessível aos responsáveis por estabelecer o plano de gestão da bacia hidrográfica, com metas de qualidade realistas e exequíveis.

\section{CONCLUSÕES}

A metodologia sugerida utiliza estatísticas simples e sua aplicação permite estabelecer VRQs para diversos descritores de qualidade da água superficial. Essa metodologia pode se constituir em uma ferramenta que pode orientar o processo decisório, facilitar o gerenciamento integrado da água e favorecer a divulgação dos resultados à sociedade.

No caso do estabelecimento de classes de uso e limites das classes, os valores sugeridos são, em sua maioria, mais restritivos em relação à Resolução CONAMA no $357 / 2005$, sendo indicador da boa qualidade da água superficial da área de estudo. Aqueles em desacordo com a legislação vigente foram fósforo, ferro e coliformes termotolerantes.

Assim, os valores limites sugeridos para as cinco classes de uso são adequados, servindo de referência aos planos de manejo de bacias hidrográficas em Santa Catarina, especialmente quando forem considerados os usos preponderantes dos recursos hídricos. Excetuando casos pontuais, o grande desafio da gestão dos recursos hídricos do estado de Santa Catarina é preservar a qualidade e a quantidade de água superficial existente.

\section{REFERÊNCIAS}

ABREU, C. H. M.; CUNHA, A. C. Qualidade da água em ecossistemas aquáticos tropicais sob impactos ambientais no Baixo Rio Jari-AP: Revisão Descritiva. Biota Amazônia, Macapá, v. 5, n. 2, p. 119-131, 2015. http://dx.doi.org/10.18561/21795746/biotaamazonia.v5n2p119-131

AGÊNCIA NACIONAL DE ÁGUAS (ANA). Atlas Esgotos: despoluição de bacias hidrográficas. Brasília: ANA, 2017. 88 p. 
AGRIZZI, D. V.; CECÍlIO, R. A.; ZANETTI, S. S.; GARCIA, G. O.; AMARAL, A. A.; FIRMINO, E. F. A.; MENDES, N. G. S. Qualidade da água de nascentes do Assentamento Paraíso. Engenharia Sanitária e Ambiental, Rio de Janeiro, v. 23, n. 3, p. 557-568, 2018. http://dx.doi.org/10.1590/s1413-41522018150701

ALVES, E. C.; SILVA, C. F.; COSSICH, E. S.; TAVARES, C. R. G.; SOUZA FILHO, E. E.; CARNIEL, A. Avaliação da qualidade da água da bacia do rio Pirapó-Maringá, Estado do Paraná, por meio de parâmetros físicos, químicos e microbiológicos. Acta Scientiarum Technology, Maringá, v. 30, n. 1, p. 39-48, 2008. https://doi.org/10.4025/actascitechnol.v30i1.3199

AMERICAN PUBLIC HEALTH ASSOCIATION (APHA). Standard Methods for the Examination of Water and Wastewater. 20 ed. Washington: American Public Health Association, American Water Woeka Association, Water Environmental Federation, 1998. 1546 p.

AMORIM, D. G.; CAVALCANTI, P. R. S.; SOARES, L. S.; AMORIM, P. E. C. Enquadramento e avaliação do índice de qualidade da água dos igarapés Rabo de Porco e Precuá, localizados na área da Refinaria Premium I, município de Bacabeira (MA). Engenharia Sanitária e Ambiental, Rio de Janeiro, v. 22, n. 2, p. 251-259, 2017. http://dx.doi.org/10.1590/s141341522016131212

ANTUNES, C. M. M.; BITTENCOURT, S. C.; RECH, T. D.; OLIVEIRA, A. C. Qualidade das águas e percepção de moradores sobre um rio urbano. Revista Brasileira de Ciências Ambientais, n. 32, p. 75-87, 2014.

ARRUDA, N. M. B.; KNOPIK, J.; SOTTOMAIOR, F. H. Avaliação da qualidade da água do Rio Tibagi antes da formação do reservatório da UHE Mauá, por meio de análise fatorial. Engenharia Sanitária e Ambiental, Rio de Janeiro, v. 22, n. 6, p. 1075-1083, 2017. http://dx.doi.org/10.1590/s1413-41522017163661

ARRUDA, N. M. B.; RIZZI, N. E.; MIRANDA, T. L. G. Análise multivariada na avaliação da qualidade de água do reservatório de Foz do Areia, Estado do Paraná. Revista Brasileira de Ciências Ambientais, n. 37, p. 26-37, 2015. http://dx.doi. org/10.5327/Z2176-947820159514

BIEGER, L.; CARVALHO, A. B. P.; STRIEDER, M. N.; MALTCHIK, L.; STENERT, C. Are the streams of the Sinos River basin of good water quality? Aquatic macroinvertebrates may answer the question. Brazilian Journal Biology, São Paulo. v. 70, n. 4, supl., p. 1207-1215, 2010. http://dx.doi.org/10.1590/S1519-69842010000600010

BOLLMANN, H. A.; MARQUES, D. Bases para a estruturação de indicadores de qualidade de águas. Revista Brasileira de Recursos Hídricos, Porto Alegre, v. 5, n. 1, p. 37-60, 2000. http://dx.doi.org/10.21168/rbrh.v5n1.p37-60

BRAGA, B.; HESPANHOL, I. Introdução à Engenharia ambiental: o desafio do desenvolvimento sustentável. 2. ed. São Paulo: Pearson Prentice Hall, 2005. 318 p.

BRASIL. Conselho Nacional do Meio Ambiente - Conama. Resolução no 357, de 17 de março de 2005. Dispõe sobre a classificação dos corpos de água e diretrizes ambientais para o seu enquadramento, bem como estabelece as condições e padrões de lançamento de efluentes, e dá outras providências. Diário Oficial da República Federativa do Brasil, Brasília, n. 053, p. 58-63, 2005.

. Conselho Nacional do Meio Ambiente - Conama. Resolução no 396, de 3 de abril de 2008. Dispõe sobre a classificação e diretrizes ambientais para o enquadramento das águas subterrâneas e dá outras providências. Diário Oficial [da] República Federativa do Brasil, Brasília, 7 abr. 2008.

Portaria do Ministério da Saúde no 2.914, de 12 de dezembro de 2011. Dispõe sobre os procedimentos de controle e de vigilância da qualidade da água para consumo humano e seu padrão de potabilidade. Diário Oficial [da] República Federativa do Brasil, Brasília, 14 dez. 2011.

BUCCI, M. H. S.; OLIVEIRA, L. F. C. Índices de Qualidade da Água e de Estado Trófico na Represa Dr. João Penido (Juiz de Fora, MG). Ambiente \& Água, Taubaté, v. 9, n. 1, p. 130-148, 2014. http://dx.doi.org/10.4136/ambi-agua.1290 
CANADIAN COUNCIL OF MINISTERS OF THE ENVIRONMENT (CCME). Canadian environmental quality guidelines: a protocol for the derivation of water quality guidelines for the protection of aquatic life. Canadá: CCME, 1999a. 37 p.

. Health Canada. Appendix XV - Protocols for deriving water quality guidelines for the protection of agricultural water uses. Canadá: CCME, 1999b. 21 p.

. Canadian environmental quality guidelines: Guidelines for Canadian recreational water quality. 3. ed. Ottawa: CCME, 1993. $161 \mathrm{p}$.

CECCONELLO, S. T.; CENTENO, L. N.; GUEDES, H. A. S. Índice de Qualidade de água modificado pela análise multivariada: estudo de caso do Arroio Pelotas, RS, Brasil. Engenharia Sanitária e Ambiental, Rio de Janeiro, v. 23, n. 5, p. 973-978, 2018. http://dx.doi.org/10.1590/s1413-41522018165394

COMPANHIA AMBIENTAL DO ESTADO DE SÃO PAULO (CETESB). Norma Técnica L5.102. Determinação de alcalinidade em águas - método da titulação potenciométrica até pH pré-determinado: método de ensaio. Cetesb, 1992a. 10 p. Disponível em: <https://cetesb.sp.gov.br/normas-tecnicas-cetesb/normas-tecnicas-vigentes/>. Acesso em: 24 out. 2019.

Norma Técnica L5.124. Determinação da dureza total em águas - método titulométrico do EDTA: método de ensaio. Cetesb, 1992b. 9 p. Disponível em: <https://www.cetesb.sp.gov.br/normas/desativadas/L5.124_ Determina\%C3\%A7\%C3\%A3o\%20da\%20dureza\%20total\%20em\%20\%C3\%A1guas\%20-\%20m\%C3\%A9todo\%20 titulometrico\%20do\%20EDTA.pdf>. Acesso em: 24 out. 2019.

COSTA, G. M.; CASSANEGO, M. B. B.; PETRY, C. T.; BENVENUTI, T.; RUBIO, M. A. K.; RODRIGUES, M. A. S.; DROSTE, A. Monitoramento químico e do potencial genotóxico para o diagnóstico da qualidade de corpos hídricos. Revista Brasileira de Ciências Ambientais, n. 32, p. 65-74, 2014.

EMPRESA DE PESQUISA AGROPECUÁRIA E EXTENSÃO RURAL DE SANTA CATARINA (EPAGRI). Relatório Síntese Qualidade de água para consumo humano e rede hídrica. Projeto Microbacia 2. Florianópolis: EPAGRI, 2005. 77 p.

GAMA, T.; GUANABARA, R.; SANTIAGO, B.; CORDEIRO, J. L. P.; OLIVEIRA, M. E. A. Avaliação conjunta das variáveis físicas e químicas do uso do solo do Rio Muriaé, Município de Itaperuna, RJ. Revista de Estudos Ambientais, Blumenau, v. 12, n. 1, p. 63-72, 2010.

GUIMARÃES, B. O.; REIS, J. A. T.; MENDONÇA, A. S. F.; AKABASSI, L. Análise probabilística de parâmetros de qualidade da água para suporte ao processo de enquadramento de cursos d'água. Engenharia Sanitária e Ambiental, Rio de Janeiro, v. 21, n. 4, p. 807-815, 2016. http://dx.doi.org/10.1590/s1413-41522016143190

MENEZES, J. P. C.; BITTENCOURT, R. P.; FARIAS, M. S.; BELLO, I. P.; FIA, R.; OLIVEIRA, L. F. C. Relação entre padrões de uso e ocupação do solo e qualidade da água em uma bacia hidrográfica urbana. Engenharia Sanitária e Ambiental, Rio de Janeiro, v. 21, n. 3, p. 519-534, 2016. http://dx.doi.org/10.1590/S1413-41522016145405

MIGLIACCIO, K. W.; HAGGARD, B. E.; CHAUBEY, I.; MATLOCK, M. Linking watershed subbasin characteristics to water quality parameters in war eagle creek watershed. American Society of Agricultural and Biological Engineers, v. 50, n. 6, p. 2007-2016, 2007. http://dx.doi.org/10.13031/2013.24104

MINELLA, J. P. G.; MERTEN, G. H. Monitoramento de bacias hidrográficas para identificar fontes de sedimentos em suspensão. Ciência Rural, Santa Maria, v. 41, n. 3, p. 424-432, 2011. http://dx.doi.org/10.1590/S010384782011000300010

MONDAL, D. K.; KAVIRAJ, A.; SAHA, S. Water Quality Parameters and Fish Biodiversity Indices as Measures of Ecological Degradation: A Case Study in Two Floodplain Lakes of India. Journal of Water Resource and Protection, Nova York, v. 2, n. 1, p. 85-92, 2010. http://dx.doi.org/10.4236/jwarp.2010.21010 
MORETTO, D. L.; PANTA, R. E.; COSTA, A. B.; LOBO, E. A. Calibration of water quality index (WQI) based on Resolution no 357/2005 of the Environmental National Council (CONAMA). Acta Limnologica Brasiliensia, Rio Claro, v. 24, n. 1, p. 29-42, 2012. http://dx.doi.org/10.1590/S2179-975X2012005000024

MOSS, B. Water pollution by agriculture. Philosophical Transaction of the Royal Society, v. 363, n. 1491, p. 659-666, 2008. https://doi.org/10.1098/rstb.2007.2176

MOZEJKO, J. Detecting and estimating trends of water quality parameters. In: VOUDOURIS, K.; VOUTSA, D., orgs. Water quality monitoring and assessment. 2012. p. 95-120. 614 p. Disponível em: <http://www.intechopen.com/ books/water-quality-monitoring-and-assessment>. Acesso em: 28 abr. 2017.

MUNIZ, D. H. F.; MORAES, A. S.; FREIRE, I. S.; CRUZ, C. J. D.; LIMA, J. E.F. W.; OLIVEIRA-FILHO, E. C. Evaluation of water quality parameters for monitoring natural, urban, and agricultural areas in the Brazilian Cerrado. Acta Limnologica Brasiliensia, Rio Claro, v. 23, n. 3, p. 307-317, 2011. http://dx.doi.org/10.1590/S2179-975X2012005000009

NONATO, E. A.; VIOLA, Z. G. G.; ALMEIDA, K. C. B.; SCHOR, H. H. R. Tratamento estatístico dos parâmetros da qualidade das águas da bacia do alto curso do rio das velhas. Química Nova, São Paulo, v. 30, n. 4, p. 797-804, 2007. http://dx.doi. org/10.1590/S0100-40422007000400008

OLIVEIRA, R. M. M.; SANTOS, E. V.; LIMA, K. C. Avaliação da qualidade da água do riacho São Caetano de Balsas (MA), com base em parâmetros físicos, químicos e microbiológicos. Engenharia Sanitária e Ambiental, Rio de Janeiro, v. 22, n. 3, p. 523-529, 2017. http://dx.doi.org/10.1590/s1413-41522017154657

PIZELLA, D. G.; SOUZA, M. P. Análise da sustentabilidade ambiental do sistema de classificação das águas doces superficiais brasileiras. Engenharia Sanitária e Ambiental, Rio de Janeiro, v. 12, n. 2, p. 139-148, 2007. http://dx.doi. org/10.1590/S1413-41522007000200005

PORTO, F. A.; PORTO, R. L. L. Gestão de Bacias Hidrográficas. Estudos Avançados, São Paulo, v. 22, n. 63, p. 43-60, 2008.

ROSSITER, K. W. L. R.; BENACHOUR, M.; MATTA, E.; MORAIA, M. M. Q. M. M.; CALADO, S. C. S.; GUNKEL, G. Diagnóstico da qualidade da água ao longo da transposição de um canal de concreto: Um estudo de caso do canal do Sertão Alagoano, Brasil. Revista Brasileira de Ciências Ambientais, n. 36, p. 145-154, 2015. https://doi.org/10.5327/Z2176947820151010

SABINO, C. V. S.; LAGE, L. V.; ALMEIDA, K. C. B. Uso de métodos estatísticos robustos na análise ambiental. Engenharia Sanitária e Ambiental, Rio de Janeiro, v. 19, n. esp., p. 87-94, 2014. http://dx.doi.org/10.1590/S141341522014019010000588

SABINO, C. V. S.; LAGE, L. V.; NORONHA, C. V. Variação sazonal e temporal da qualidade das águas em um ponto do córrego Gameleiras usando técnicas quimiométricas robustas. Engenharia Sanitária e Ambiental, Rio de Janeiro, v. 22, n. 5, p. 969-983, 2017. http://dx.doi.org/10.1590/s1413-41522017158455

SANTA CATARINA (Estado). Decreto no 14.250, de 5 de junho de 1981. Regulamenta dispositivos da Lei no 5.793, de 15 de outubro de 1980, referentes à Proteção e a Melhoria da Qualidade Ambiental. Diário Oficial [do] Estado de Santa Catarina, Florianópolis, 9 jun. 1981.

SIQUEIRA, G. W.; APRILE, F.; MIGUÉIS, A. M. Diagnóstico da qualidade da água do rio Parauapebas (Pará-Brasil). Acta Amazonica, Manaus, v. 42, n. 3, p. 413-422, 2012. http://dx.doi.org/10.1590/S0044-59672012000300014

SMITH, R. A.; ALEXANDER, R. B.; SCHWARZ, G. E. Natural background concentrations of nutrients in streams and rivers of the Conterminous United States. Environmental Science and Technology, v. 37, n. 14, p. 3039-3047, 2003. https://doi.org/10.1021/es020663b 
SOUZA, A. P. Índice de qualidade da água em microbacias hidrográficas com diferentes coberturas vegetais, no sul do Estado do Espírito Santo. 71f. Dissertação (Mestrado) - Universidade Federal do Espírito Santo, Jerônimo Monteiro, 2011.

STROBL, R. O.; ROBILLARD, P. D. Network design for water quality monitoring of surface freshwaters: a review. Journal of Environmental Management, v. 87, n. 4, p. 639-648, 2008. https://doi.org/10.1016/j.jenvman.2007.03.001

TRINDADE, A. L. C.; ALMEIDA, K. C. B.; BARBOSA, P. E.; OLIVEIRA, S. M. A. C. Tendências temporais e espaciais da qualidade das águas superficiais da sub-bacia do Rio das Velhas, estado de Minas Gerais. Engenharia Sanitária $e$ Ambiental, Rio de Janeiro, v. 22, n. 1, p. 13-24, 2017. http://dx.doi.org/10.1590/s1413-41522016131457

UNITED STATES ENVIRONMENTAL PROTECTION AGENCY (USEPA). Aquatic life ambient water quality criteria for Ammonia - freshwater. Washington, D.C.: USEPA, 2013. 242 p.

. Quality Criteria for water. Washington, D.C., USEPA, 1986. 450 p.

VON SPERLING, M.; CHERNICHARO, C. A. L. Urban wastewater treatment Technologies and implementation of discharge standards in developing countries. Urban Water, v. 4, n. 1, p. 105-114, 2002. https://doi.org/10.1016/S14620758(01)00066-8

WORLD HEALTH ORGANIZATION (WHO). Guidelines for Drinking-water Quality. 3. ed. Genebra: WHO, 2011. v. 1. 541 p.

ZUCCO, E.; PINHEIRO, A.; SOARES, P. A.; DESCHAMPS, F. C. Qualidade das águas em uma bacia agrícola: subsídios ao programa de monitoramento. Revista de Estudos Ambientais, Blumenau, v. 14, n. 3, p. 88-97, 2012. http://dx.doi. org/10.7867/1983-1501.2012v14n3p88-97 